\title{
Shape, Motion, and Parameter Estimation of Flexible Space Structures using Laser Rangefinders
}

\author{
Matthew D. Lichter ${ }^{*}$, Steven Dubowsky ${ }^{*}$, Hiroshi Ueno ${ }^{* *}$, and Shinji Mitani ${ }^{* *}$ \\ ${ }^{*}$ Department of Mechanical Engineering \\ ** Japan Aerospace Exploration Agency \\ Massachusetts Institute of Technology \\ Cambridge, MA, USA \\ \{lichter |dubowsky\}@mit.edu \\ Guidance, Control, and Dynamics Group \\ Tsukuba, Ibaraki, Japan \\ \{ueno.hiroshi|mitani.shinji\}@jaxa.jp
}

\begin{abstract}
Future space missions are expected to use robotic systems to assemble, inspect, and maintain large space structures in orbit. For effective planning and control, robots must know the deformations and motions of the structures with which they interact. This paper presents a method for estimating the shape, motion, and dynamic model parameters of a vibrating space structure using asynchronous raster-scanning range imagers. The method assumes that the mode shapes are approximately known a priori. A Kalman filter exploits a mechanics-based dynamic model to extract the modal frequencies and damping as well as the modal coefficients and their time rate of change. Theoretical development and experimental results using emulated space hardware are presented.
\end{abstract}

Index Terms - space structure, laser rangefinder, shape estimation, motion estimation, cooperative sensing.

\section{INTRODUCTION}

Future space missions are expected to use autonomous robotic systems to assemble, inspect, and maintain large space structures in orbit [1][2][3]. Examples include the International Space Station, large synthetic aperture telescopes, and space solar power systems [4][5]. To safely plan and execute tasks, robotic systems require knowledge of structural deformations and motions. Remote sensing and estimation of target dynamics and model parameters will be a fundamental challenge for these missions.

Many researchers have used embedded sensors such as strain gauges and accelerometers to directly measure the motions and deformations of flexible structures [6][7][8]. However, the hardware costs and the complexity of this approach may be prohibitive for very large space structures that span hundreds or thousands of meters [9]. An alternative approach is to use range imaging sensors that may be available from free-flying robotic workers (see Fig. 1). Such sensors might include stereo cameras [10] or laser rangefinders [11]. However, there are a number of challenges to this approach. Range images can be highly noisy and data may be missing from many areas of the structure due to the harsh lighting conditions found in space. Strong sunlight,

This work is sponsored by the Japan Aerospace Exploration Agency (JAXA) and the Japan Society for Promotion of Science (JSPS) Postdoctoral Fellowship. high-contrast scenes, and reflective materials (e.g. solar panels and metallic foils found on spacecraft) pose significant challenges to many image processing algorithms [12]. Tracking specific points on the structure can be very difficult as the lighting or sensor positions change. Complicating the problem is the fact that computational resources tend to be very limited in space-qualified hardware.

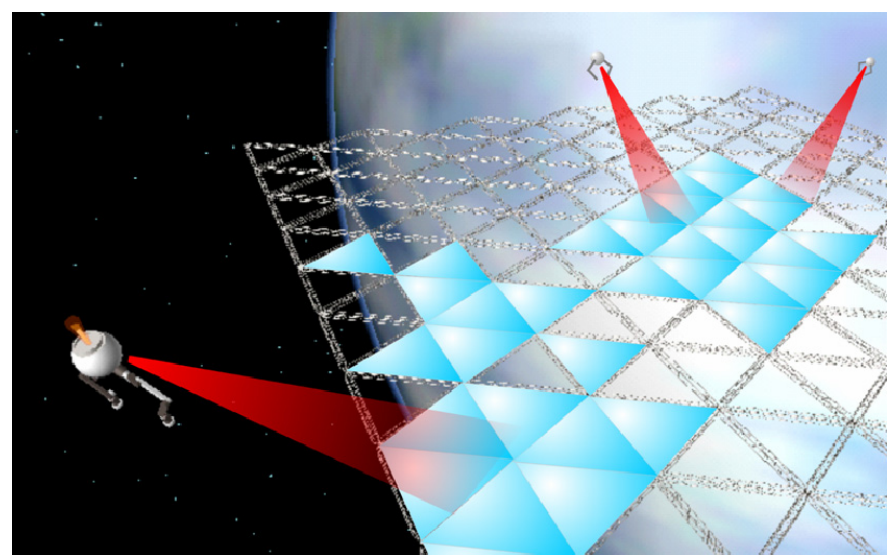

Fig. 1. Using onboard vision sensors to estimate structural information.

This paper presents a method for estimating the shape and motion of a region of interest on a flexible space structure, using data gathered from one or more raster-scanning range sensors. This method takes advantage of a key feature of the application, which is that the dynamics of systems in space are highly deterministic and can be modeled accurately. This allows a method that does not require the tracking of structural features over time. Further, it yields an estimator that is computationally efficient while being robust to substantial sensor noise and a priori uncertainty.

This paper builds upon the authors' previous works $[13][14]$ in two ways. First, it presents substantial new experimental support for the method, which was studied largely in simulation previously. Second, this paper broadens the theoretical foundation so that raster scanning types of sensors can be used. Previous works dealt strictly with sensors that capture range points synchronously, such as stereo vision systems. However, many important range imaging technologies, such as laser striping systems, employ raster scanning and hence capture points asynchronously. The coupling of the spatial and time domains by the sensor adds 
substantial complexity to the estimation problem and is considered for the first time here.

\section{GENERAL APPROACH}

\section{A. Assumptions}

Sensors are assumed to provide discrete 3-D range image clouds of the structure at known intervals. The time at which each point in the cloud is acquired is assumed to be known. Sensor noise may be substantial and is assumed to be additive, white, and unbiased, but not necessarily Gaussian. If multiple cooperative sensors are used to gather range images, their relative poses are assumed to be accurately known so that their data can be expressed in a common reference frame.

The structural dynamics are assumed to be linear or weakly nonlinear. The modal coefficients $A(t)$ and their time rate of change $V(t)$ are assumed to approximately take the form of decaying sinusoids:

$$
\begin{aligned}
& A_{i}(t) \approx e^{-\alpha_{i} t} \sin \left(\omega_{i} t+\varphi_{i}\right) \\
& V_{i}(t) \equiv \frac{d}{d t} A_{i}(t) \approx e^{-\alpha_{i} t} \omega_{i} \cos \left(\omega_{i} t+\varphi_{i}\right)-\alpha_{i} A_{i}(t)
\end{aligned}
$$

The structure's mode shapes are assumed to be reasonably well-known to the estimator a priori. These could be provided from theoretical or empirical analysis performed offline beforehand. Modal information is assumed to be updated whenever the fundamental mode shapes change (e.g. due to a structural configuration change, added mass, etc.). The modal frequencies are assumed to be known to within \pm 20 percent a priori.

\section{B. Approach}

Let the natural mode shapes of the structure be denoted as $\Phi_{i}(x)$ for each mode $i$. For a linear elastic system, the dynamic response of the structural deformations $z(x, t)$ can be written as

$$
z(x, t)=\sum_{i=1}^{m} A_{i}(t) \Phi_{i}(x)=A(t)^{T} \Phi(x)
$$

where $z(x, t)$ is the deflection from the structure's equilibrium state, $m$ is the number of modes excited in the response, and $A_{i}(t)$ is the $i^{\text {th }}$ modal coefficient, which oscillates sinusoidally according to (1).

The goal is to estimate the time domain functions $A_{i}(t)$ for all modes of interest. This will reduce shape estimation to simply a modal reconstruction using the estimates of $A_{i}(t)$ and the mode shapes $\Phi_{i}(x)$.

Estimation of $A_{i}(t)$ will occur here in two steps. First, a modal decomposition in the spatial domain is performed on the range image to arrive at a coarse estimate $\breve{A}_{i}(t)$. This estimate is then filtered in the time domain using a Kalman filter to arrive at a refined estimate $\hat{A}_{i}(t)$. Note the hat notation used to denote coarse and refined estimates.

\section{MODAL DECOMPOSITION}

The estimation process first uses a modal decomposition of the visual data to find coarse estimates of the modal coefficients $A(t)$.

Define an inner product (dot product) over some space $X$ as

$$
\langle a, b\rangle_{X} \equiv \int_{X} a(x) b^{T}(x) d x=\int_{X}\left[\begin{array}{ccc}
a_{1}(x) b_{1}(x) & a_{1}(x) b_{2}(x) & \cdots \\
a_{2}(x) b_{1}(x) & a_{2}(x) b_{2}(x) & \\
\vdots & & \ddots
\end{array}\right] d x .
$$

Let the space $X$ be the "backbone" surface of the target structure. For example, if the structure is a planar sheet of uniform thickness, then the space $X$ is the 2-D reference surface embedded in the sheet at its equilibrium configuration, and $z(x, t)$ represents the deformation normal to the surface at some location $x$ in $X$. One useful property of mode shapes is that they are orthogonal in the space $X$ (i.e. $\left\langle\Phi_{i}, \Phi_{j}\right\rangle_{X}=0$ for all $i \neq j)$.

Consider a subspace $Y \subset X$, which represents a discrete, not necessarily uniform sampling of the space $X$ (see Fig. 2). The subspace $Y$ is the sample space defined by the range measurements, and may be changing in time as the sensor and/or structure move.

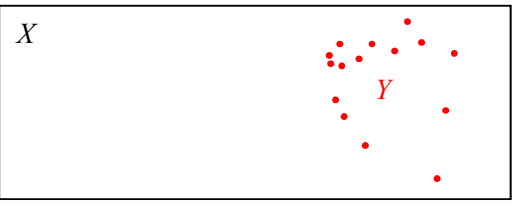

Fig. 2. Sample space $Y$ in complete space $X$.

In this discrete space the inner product reduces to:

$$
\langle a, b\rangle_{Y}=\sum_{k=1}^{n} a\left(y_{k}\right) b^{T}\left(y_{k}\right)
$$

where $y_{k}$ is the $k^{\text {th }}$ discrete point in the sample space $Y$ and $n$ is the number of discrete points in the sample space.

Define a symmetric modal correlation matrix $M_{Y}$ to describe the inner products of the mode shapes in the sample space $Y$, for the $m$ excited modes:

$$
M_{Y} \equiv\langle\Phi, \Phi\rangle_{Y}=\left[\begin{array}{ccccc}
\left\langle\Phi_{1}, \Phi_{1}\right\rangle_{Y} & \cdots & \left\langle\Phi_{1}, \Phi_{i}\right\rangle_{Y} & \cdots & \left\langle\Phi_{1}, \Phi_{m}\right\rangle_{Y} \\
\vdots & \ddots & \vdots & \ddots & \vdots \\
\left\langle\Phi_{i}, \Phi_{1}\right\rangle_{Y} & \cdots & \left\langle\Phi_{i}, \Phi_{i}\right\rangle_{Y} & \cdots & \left\langle\Phi_{i}, \Phi_{m}\right\rangle_{Y} \\
\vdots & \ddots & \vdots & \ddots & \vdots \\
\left\langle\Phi_{m}, \Phi_{1}\right\rangle_{Y} & \cdots & \left\langle\Phi_{m}, \Phi_{i}\right\rangle_{Y} & \cdots & \left\langle\Phi_{m}, \Phi_{m}\right\rangle_{Y}
\end{array}\right] .
$$

If $Y$ is dense and uniformly distributed over $X$, then $M_{Y} \approx \lambda M_{X}$ for some scale factor $\lambda$. However, this paper considers the general case in which the sample space $Y$ is not a uniform and complete sampling of the complete structure space $X$ (e.g. the sensors observe only a portion of the 
structure). If the sample space is changing (e.g. the sensors are moving), the modal correlation matrix $M_{Y}$ and the inner product operator $\langle a, b\rangle_{Y}$ are not constant and must be recomputed at each time step.

By the Cauchy-Schwarz inequality, it can be shown that $M_{Y}$ is positive semidefinite [13]. The semidefinite condition arises only from a pathological choice of the sample space $Y$ such that certain modes are unobservable or undiscernable (i.e. the modes are aliased spatially). The condition number of $M_{Y}$ can be checked to determine the proximity to this condition. All further discussion assumes that $M_{Y}$ is positive definite, well-conditioned, and invertible.

In this problem, a vector of range image points is provided to the estimator at some reference time $t$. The $k^{\text {th }}$ element of the vector (the $k^{\text {th }}$ point) measures the displacement of the structure at some location $y_{k}$ and time $t+\Delta_{k}$, where $\Delta_{k}$ is a known time delay due to the asynchronous nature of raster scanning. The $k^{\text {th }}$ measurement can be written as

$$
\begin{aligned}
\bar{z}_{k}(t) & =\left(\sum_{i=1}^{m} A_{i}\left(t+\Delta_{k}\right) \Phi_{i}\left(y_{k}\right)\right)+e_{k} \\
& =A\left(t+\Delta_{k}\right)^{T} \Phi\left(y_{k}\right)+e_{k}
\end{aligned}
$$

where the overbar is used to denote a measurement, $A\left(t+\Delta_{k}\right)$ is the vector of modal coefficients at time $t+\Delta_{k}$, $\Phi\left(y_{k}\right)$ is the vector of mode shapes evaluated at location $y_{k}$, and $e_{k}$ is additive sensor noise. The delay $\Delta_{k}$ is a significant complication to the original method presented in [13][14] and methods to handle it are now presented.

Applying trigonometric substitutions to (1) yields

$$
A_{i}\left(t+\Delta_{k}\right)=A_{i}(t) f_{i}\left(\Delta_{k}\right)+V_{i}(t) g_{i}\left(\Delta_{k}\right)
$$

where $f_{i}$ and $g_{i}$ are delay modulation functions given by

$$
\begin{aligned}
& f_{i}\left(\Delta_{k}\right)=e^{-\alpha_{i} \Delta_{k}}\left(\cos \left(\omega_{i} \Delta_{k}\right)+\frac{\alpha_{i} \sin \left(\omega_{i} \Delta_{k}\right)}{\omega_{i}}\right) \\
& g_{i}\left(\Delta_{k}\right)=e^{-\alpha_{i} \Delta_{k}}\left(\frac{\sin \left(\omega_{i} \Delta_{k}\right)}{\omega_{i}}\right)
\end{aligned}
$$

Reasonable a priori guesses should be used for the frequency and damping terms in (6). In this application, damping $\alpha_{i}$ and time delay $\Delta_{k}$ are usually small enough that $\alpha_{i}$ can be set to zero in (6) without noticeably affecting estimator performance.

Substituting (5) into (4) yields

$$
\begin{aligned}
\bar{z}_{k}(t) & =\sum_{i=1}^{m} A_{i}(t) f_{i}\left(\Delta_{k}\right) \Phi_{i}\left(y_{k}\right) \\
& +\sum_{i=1}^{m} V_{i}(t) g_{i}\left(\Delta_{k}\right) \Phi_{i}\left(y_{k}\right)+e_{k}
\end{aligned}
$$

$$
\begin{gathered}
\Phi_{i}^{f}\left(y_{k}, \Delta_{k}\right) \equiv f_{i}\left(\Delta_{k}\right) \Phi_{i}\left(y_{k}\right) \\
\Phi_{i}^{g}\left(y_{k}, \Delta_{k}\right) \equiv g_{i}\left(\Delta_{k}\right) \Phi_{i}\left(y_{k}\right)
\end{gathered}
$$

These can be viewed as fundamental mode shapes in the space-time domain (as opposed to the original modes $\Phi_{i}\left(y_{k}\right)$, which reside strictly in the spatial domain). Equation (7) can then be written in the compact form

$$
\begin{aligned}
\bar{z}_{k}(t) & =A(t)^{T} \Phi^{f}\left(y_{k}, \Delta_{k}\right)+V(t)^{T} \Phi^{g}\left(y_{k}, \Delta_{k}\right)+e_{k} \\
& =\left\{\begin{array}{l}
A(t) \\
V(t)
\end{array}\right\}{ }^{T}\left\{\begin{array}{l}
\Phi^{f}\left(y_{k}, \Delta_{k}\right) \\
\Phi^{g}\left(y_{k}, \Delta_{k}\right)
\end{array}\right\}+e_{k}
\end{aligned}
$$

Define a delay-modulated modal correlation matrix $M_{Y}^{\prime}$ using the delay-modulated mode shapes:

$$
\begin{gathered}
M_{Y}^{\prime} \equiv\left[\begin{array}{cccc}
\left\langle\Phi^{f}, \Phi^{f}\right\rangle_{Y} & \left\langle\Phi^{f}, \Phi^{g}\right\rangle_{Y} \\
\left\langle\Phi^{g}, \Phi^{f}\right\rangle_{Y} & \left\langle\Phi^{g}, \Phi^{g}\right\rangle_{Y}
\end{array}\right] \\
=\left[\begin{array}{cccccc}
\left\langle\Phi_{1}^{f}, \Phi_{1}^{f}\right\rangle_{Y} & \cdots & \left\langle\Phi_{1}^{f}, \Phi_{m}^{f}\right\rangle_{Y} & \left\langle\Phi_{1}^{f}, \Phi_{1}^{g}\right\rangle_{Y} & \cdots & \left\langle\Phi_{1}^{f}, \Phi_{m}^{g}\right\rangle_{Y} \\
\vdots & \ddots & \vdots & \vdots & \ddots & \vdots \\
\left\langle\Phi_{m}^{f}, \Phi_{1}^{f}\right\rangle_{Y} & \cdots & \left\langle\Phi_{m}^{f}, \Phi_{m}^{f}\right\rangle_{Y} & \left\langle\Phi_{m}^{f}, \Phi_{1}^{g}\right\rangle_{Y} & \cdots & \left\langle\Phi_{m}^{f}, \Phi_{m}^{g}\right\rangle_{Y} \\
\left\langle\Phi_{1}^{g}, \Phi_{1}^{f}\right\rangle_{Y} & \cdots & \left\langle\Phi_{1}^{g}, \Phi_{m}^{f}\right\rangle_{Y} & \left\langle\Phi_{1}^{g}, \Phi_{1}^{g}\right\rangle_{Y} & \cdots & \left\langle\Phi_{1}^{g}, \Phi_{m}^{g}\right\rangle_{Y} \\
\vdots & \ddots & \vdots & \vdots & \ddots & \vdots \\
\left\langle\Phi_{m}^{g}, \Phi_{1}^{f}\right\rangle_{Y} & \cdots & \left\langle\Phi_{m}^{g}, \Phi_{m}^{f}\right\rangle_{Y} & \left\langle\Phi_{m}^{g}, \Phi_{1}^{g}\right\rangle_{Y} & \cdots & \left\langle\Phi_{m}^{g}, \Phi_{m}^{g}\right\rangle_{Y}
\end{array}\right]
\end{gathered}
$$

Taking inner products of the measurements with the delaymodulated mode shapes yields

$$
\begin{aligned}
\left\{\begin{array}{c}
\left\langle\Phi^{f}, \bar{z}\right\rangle_{Y} \\
\left\langle\Phi^{g}, \bar{z}\right\rangle_{Y}
\end{array}\right\} & =\left\{\begin{array}{c}
\left\langle\Phi_{1}^{f}, \bar{z}\right\rangle_{Y} \\
\vdots \\
\left\langle\Phi_{m}^{f}, \bar{z}\right\rangle_{Y} \\
\left\langle\Phi_{1}^{g}, \bar{z}\right\rangle_{Y} \\
\vdots \\
\left\langle\Phi_{m}^{g}, \bar{z}\right\rangle_{Y}
\end{array}\right\} \\
& =M_{Y}^{\prime}\left\{\begin{array}{c}
A(t) \\
V(t)
\end{array}\right\}+\left\{\begin{array}{c}
\left\langle\Phi^{f}, e\right\rangle_{Y} \\
\left\langle\Phi^{g}, e\right\rangle_{Y}
\end{array}\right\}
\end{aligned}
$$

Rearranging yields

$$
M_{Y}^{\prime-1}\left\{\begin{array}{l}
\left\langle\Phi^{f}, \bar{z}\right\rangle_{Y} \\
\left\langle\Phi^{g}, \bar{z}\right\rangle_{Y}
\end{array}\right\}=\left\{\begin{array}{l}
A(t) \\
V(t)
\end{array}\right\}+M_{Y}^{\prime-1}\left\{\begin{array}{l}
\left\langle\Phi^{f}, e\right\rangle_{Y} \\
\left\langle\Phi^{g}, e\right\rangle_{Y}
\end{array}\right\}
$$

and therefore an unbiased estimate of $A(t)$ and $V(t)$ is given by

Define the delay-modulated mode shapes 


$$
\begin{aligned}
\left\{\begin{array}{l}
\breve{A}(t) \\
\vec{V}(t)
\end{array}\right\} & =M_{Y}^{\prime-1}\left\{\begin{array}{l}
\left\langle\Phi^{f}, \bar{z}\right\rangle_{Y} \\
\left\langle\Phi^{g}, \bar{z}\right\rangle_{Y}
\end{array}\right\} \\
& =\left\{\begin{array}{l}
A(t) \\
V(t)
\end{array}\right\}+w
\end{aligned}
$$

where the error $w$ is given by

$$
w \equiv\left\{\begin{array}{l}
\breve{A}(t)-A(t) \\
\breve{V}(t)-V(t)
\end{array}\right\}=M_{Y}^{\prime-1}\left\{\begin{array}{l}
\left\langle\Phi^{f}, e\right\rangle_{Y} \\
\left\langle\Phi^{g}, e\right\rangle_{Y}
\end{array}\right\} .
$$

The error covariance on the estimate given by (12) is therefore

$$
\begin{gathered}
\Lambda_{w w} \equiv E\left[w \cdot w^{T}\right] \\
=M_{Y}^{\prime-1} E\left(\left[\frac{\left\langle\Phi^{f}, e\right\rangle_{Y}\left\langle e, \Phi^{f}\right\rangle_{Y} \mid\left\langle\Phi^{f}, e\right\rangle_{Y}\left\langle e, \Phi^{g}\right\rangle_{Y}}{\left\langle\left\langle\Phi^{g}, e\right\rangle_{Y}\left\langle e, \Phi^{f}\right\rangle_{Y}\right|\left\langle\Phi^{g}, e\right\rangle_{Y}\left\langle e, \Phi^{g}\right\rangle_{Y}}\right]\right) M_{Y}^{\prime-1} .
\end{gathered}
$$

If the variance on the noise $e$ is approximately the same for all image points, this reduces to

$$
\Lambda_{w w}=\sigma_{e}^{2} M_{Y}^{\prime-1}
$$

where $\sigma_{e}{ }^{2} \equiv E\left[e_{k}^{2}\right]$. If the noise variance is substantially different for each range image point, the equation is not as concise but $\Lambda_{w w}$ is still easily solved [13].

Equation (12) represents an easily computed coarse estimate of $A(t)$ and $V(t)$ that is unbiased and has a Gaussian error distribution with statistics computed from (13). It is a minimum-least-square-error estimate of $A(t)$ and $V(t)$ using data from a single sample time.

\section{KALMAN FILTERING: SINUSOID ESTIMATION}

A Kalman filter is used to observe the time series $\breve{A}(t)$ and $\breve{V}(t)$ and extract a better estimate of $A(t)$ and $V(t)$ using knowledge that they are weakly decaying sinusoids. Observation over time also allows the estimation of modal parameters.

The estimated state consists of $\hat{A}(t)$, its time rate of change $\hat{V}(t)$, the natural frequencies $\hat{\omega}$, and the modal damping rate $\hat{\alpha}$. If the true modal coefficients follow the trajectory given by (1), differentiation and substitution leads to the discretetime process model

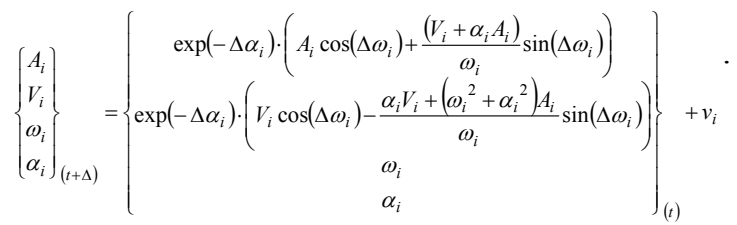

Note the lack of dependence on phase $\varphi_{i}$. If known external forces are applied to the structure (e.g. from robotic systems), they should be incorporated into the process model here.

Process noise is indicated in (14) by $v \equiv\left\{\begin{array}{llll}v_{A} & v_{V} & v_{\omega} & v_{\alpha}\end{array}\right\}^{T}$, and is characterized by the covariance matrix

$$
\Lambda_{v v} \equiv E\left[v v^{T}\right] .
$$

The values in this matrix should be chosen to describe the uncertainty in the dynamic model due to unmodeled disturbances and parameter uncertainty.

The Kalman filter measurement model is given by (12). Measurement noise $w$ is white, unbiased, and Gaussian with statistics computed from (13).

The initial a posteriori state estimate is given by

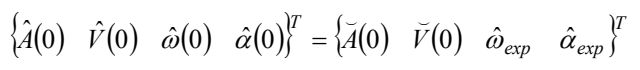

where $\omega_{\exp }$ and $\alpha_{\text {exp }}$ are the expected frequencies and damping predicted offline beforehand.

The initial a posteriori state error covariance should be chosen to describe the uncertainty in (16). The covariance on $\hat{A}(0)$ and $\hat{V}(0)$ will be the measurement covariance given by (13) while the other portions of the state covariance must be based engineering judgment of the actual system.

The implementation of the Kalman filter is straightforward using (12-16). Since the process model is nonlinear, an extended Kalman filter, unscented Kalman filter, particle filter, or a more general form of Bayesian estimator must be used. The unscented Kalman filter [15] was used here with good observed performance.

\section{Shape Estimation: Modal Reconstruction}

Shape estimation is simply a modal reconstruction using (2) and the estimated modal coefficients:

$$
\hat{z}(x, t)=\sum_{i=1}^{m} \hat{A}_{i}(t) \hat{\Phi}_{i}(x)=\hat{A}(t)^{T} \hat{\Phi}(x) .
$$

The hat notation is used on the mode shapes $\Phi(x)$ as a reminder that the estimator might not know these perfectly, as they are based on theoretical analyses performed offline.

If mode shape knowledge is perfect, the uncertainty in the shape estimate is given by

$$
\begin{aligned}
\Lambda_{z z}(x, t) & \equiv E\left[(\hat{z}(x, t)-z(x, t))(\hat{z}(x, t)-z(x, t))^{T}\right] \\
& =\Phi(x)^{T} \Lambda_{\hat{A} \hat{A}}(t) \Phi(x)
\end{aligned}
$$

where $\Lambda_{\hat{A} \hat{A}}(t)$ is the error covariance on the estimate $\hat{A}(t)$. Analysis suggests that mode shape and modal parameter uncertainty degrades estimator performance gracefully rather 
than catastrophically [13], however a full discussion of this topic is beyond the scope of this paper.

\section{EXPERIMENTAL RESULTS}

Experiments were conducted using representative structures and sensors to evaluate the performance of the estimation algorithm. A flexible panel measuring $2.5 \mathrm{~m}$ long and $0.5 \mathrm{~m}$ wide was mounted on air bearings and placed on a highly polished air table as shown in Figs. 3 and 4. One end of the structure is rigidly constrained to ground. Structural vibrations occur in the horizontal plane orthogonal to the gravitation vector. The periods of vibration for the first five modes are approximately $44,6.8,2.6,1.4$, and $0.55 \mathrm{~s}$, respectively. This system emulates a space structure reasonably well since it possesses low damping and will sustain vibrations in several modes for many cycles before damping out.

The dynamics of this system can be represented using the Bernoulli-Euler cantilever beam model. Here, the analytical solutions for the mode shapes were used in the estimator, although any method of mode shape determination could have been used (finite element analysis, empirical measurement of a scale model, etc.). Initial guesses for the modal frequencies were taken from very rough (one digit precision) measurements using a stopwatch. Certainly, more accurate a priori estimates of the mode shapes and parameters could have been used; however, these coarse methods were used to demonstrate the estimator's robustness to a priori uncertainty.

For these experiments, a Pulstec Industrial Co., TDS-series 3D laser scanner was used to observe the structure. The scanner was mounted on a robotic manipulator to allow accurate repositioning between experiments (see Fig. 4). The scanner has a field of view of approximately 8 degrees by 7 degrees and typically observes only 5 to 30 percent of the structure's surface area during an experiment. Range measurement noise on the sensor is on the order of $20 \mathrm{~mm}$ at a range of $2 \mathrm{~m}$ (the approximate distance used in these experiments).

The system takes two scans per second, with even scans sweeping upward through space and odd scans sweeping downward. During each scan, the system gathers approximately 2000 points, distributed over a 400 -ms interval. That is, the last point in each scan is captured approximately $400 \mathrm{~ms}$ after the first point. Prior to conducting experiments, the time stamps for all points (i.e. the $\Delta_{k}$ values in (4) for all $k$ ) were recorded and provided to the estimation algorithm.

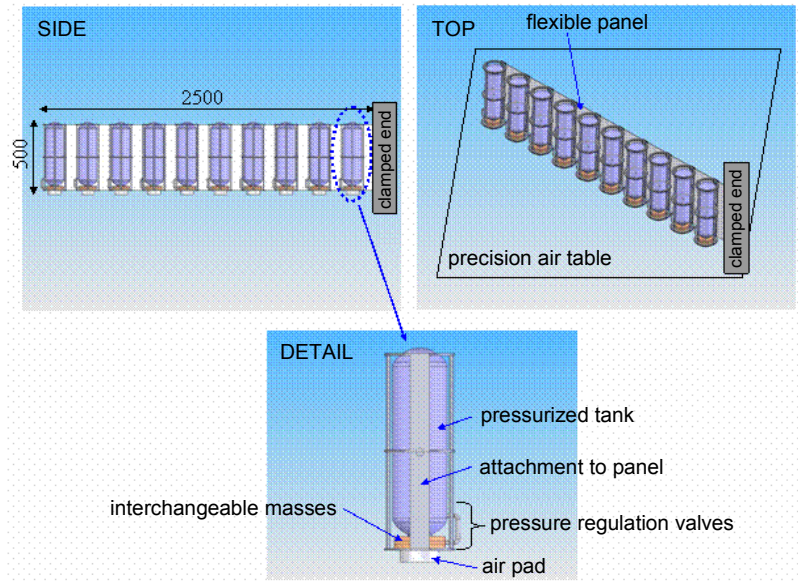

Fig. 3. Schematic of experimental system.

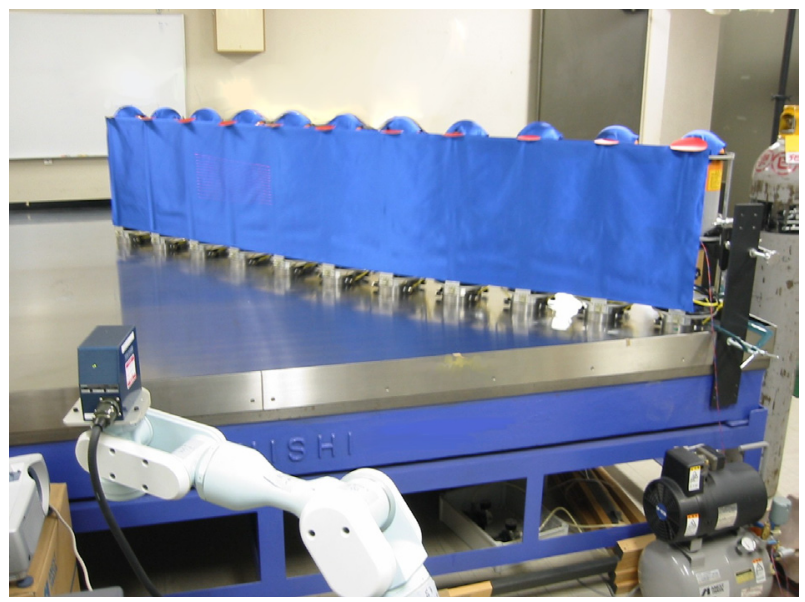

Fig. 4. Photo of experimental system. Laser rangefinder mounted on manipulator arm can be seen in the foreground at left.

Fig. 5 shows typical experimental results for the coarse estimates $\breve{A}(t)$ and the Kalman-filtered estimates $\hat{A}(t)$ for several modes of vibration. Note that the Kalman filter performs effectively like a notch filter and removes spurious signals and bias from the coarse estimates.

Figs. 6 and 7 show typical experimental results for the estimation of modal frequencies and damping. Frequency estimates converged on the order of two periods of the mode being estimated. Here, damping has a comparatively small influence on system dynamics and therefore can only be observed over long time intervals, which explains the relatively slow convergence of these parameter estimates. It should be noted that the observability of parameters declines over time in a damped system such as this. Many of the higher modes damped out too quickly to be estimated with reasonable confidence. Table 1 summarizes the mean and standard deviation of the final parameter estimates for the first three modes, recorded over fifteen independent trials. The frequency values are consistent with direct empirical measurements of the structure. It was difficult to obtain a consistent baseline measurement of damping, however, since it varied somewhat throughout trials due to uncontrollable environmental factors. 

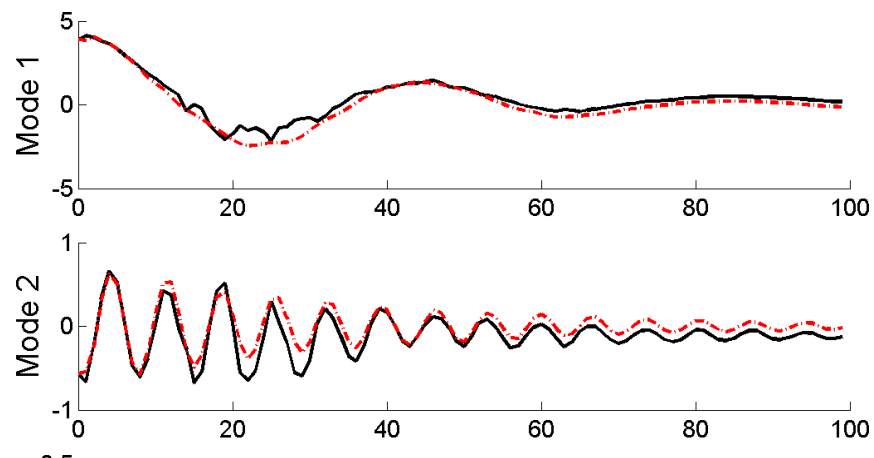

0.5

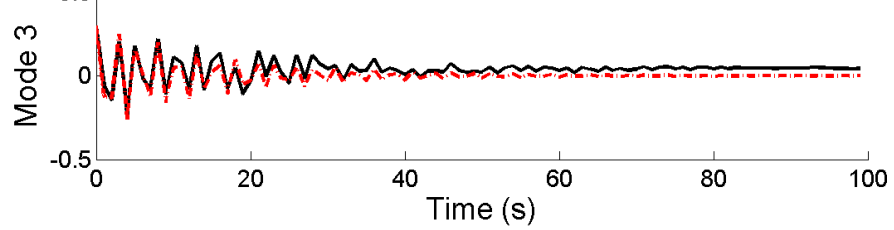

Fig. 5. Coarse estimates of modal coefficients (solid) with Kalman-filtered estimates superimposed (dashed) (experimental results).

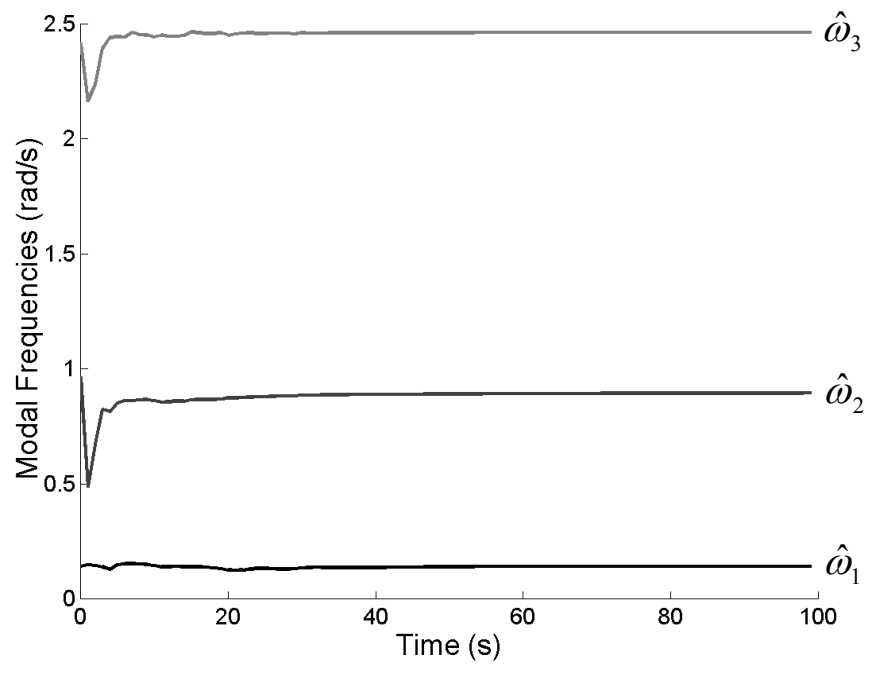

Fig. 6. Modal frequency estimates (experimental results).

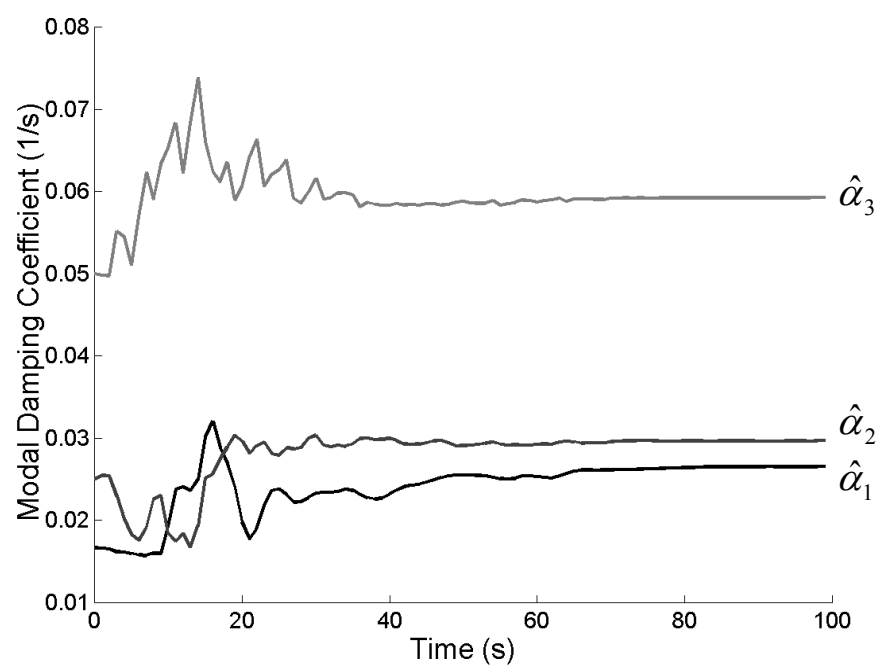

Fig. 7. Modal damping estimates (experimental results).
TABLE 1. PARAMETER ESTIMATES AFTER 100 SECONDS OF OBSERVATION, FOR FIFTEEN INDEPENDENT TRIALS.

\begin{tabular}{|c|c|c|}
\hline $\begin{array}{c}\text { mode } \\
\text { number }\end{array}$ & $\omega$ estimate $(\mathrm{rad} / \mathrm{s})$ & $\alpha$ estimate $(1 / \mathrm{s})$ \\
\hline 1 & $0.14 \pm 0.016$ & $0.033 \pm 0.012$ \\
2 & $0.87 \pm 0.025$ & $0.070 \pm 0.036$ \\
3 & $2.44 \pm 0.33$ & $0.146 \pm 0.091$ \\
\hline
\end{tabular}

The goal of estimation is more than simply observing structural deformations and parameters, however. Knowledge of the dynamic model and the ability to estimate parameters allows the estimator to predict future motions and shape of the structure. This predictive capacity is useful and sometimes necessary for certain planning and control tasks such as robotic rendezvous and docking. Fig. 8 illustrates the estimated motions of the structure under two different situations. The solid line represents the best estimate the filter can achieve, incorporating all measurements from time $t=0 \ldots 100$. By contrast, the dashed line shows the estimate if the filter receives measurements only during time $t=0 \ldots 25$ and then predicts the motion for $t=25 \ldots 100$ by extrapolating its last estimate. The predictions can be seen to track the actual motions reasonably well for some time after measurements are stopped. Frequency, phase, amplitude, and damping appear to be estimated reasonably accurately by the time the last measurement is processed. Over time, phase error accumulates due to small errors in the frequency estimate, and eventually the prediction loses synchronization with the actual motions.
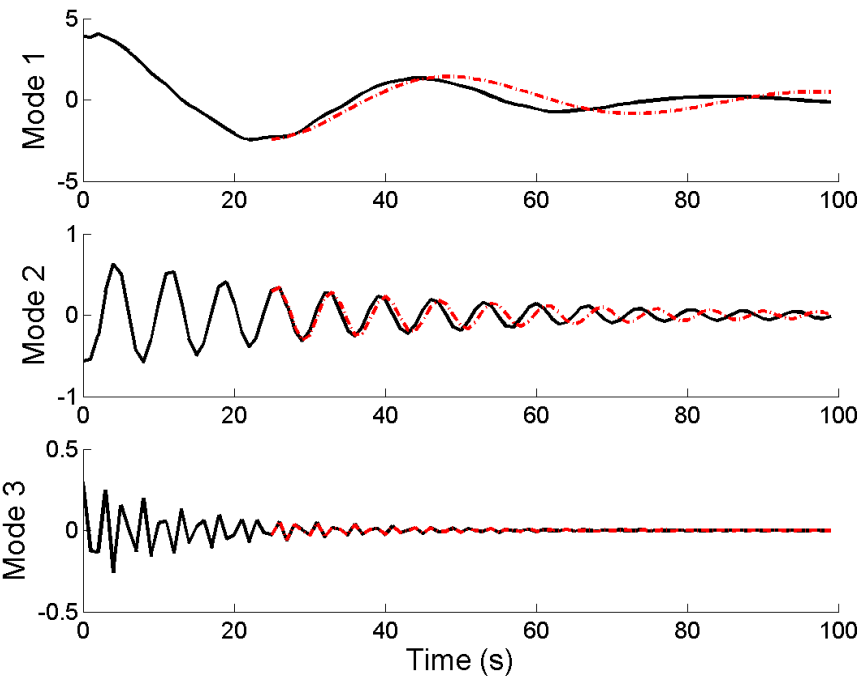

Fig. 8. Coefficient predictions when measurements are halted at time $t=25$ (dashed). Solid lines effectively represent the actual coefficients, found by using all measurements through time $t=100$. 

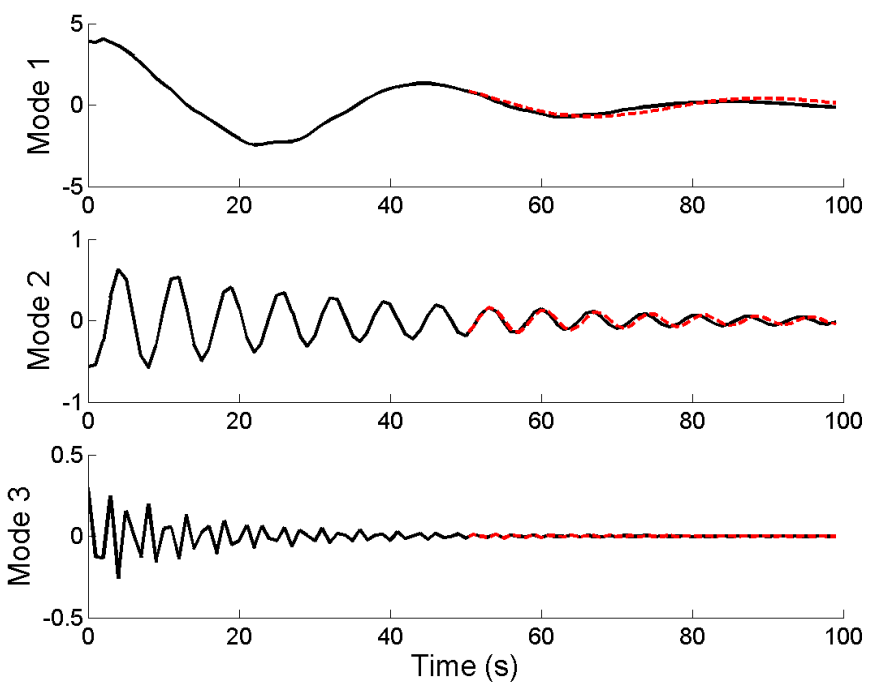

Fig. 9. Coefficient predictions when measurements are halted at time $t=50$ (dashed). Solid lines effectively represent the actual coefficients, found by using all measurements through time $t=100$.

As one might expect, if the estimator is given more measurements before it begins predicting motion, its parameter estimates will be more accurate and thus motion predictions will track the actual system for a greater length of time. This is illustrated in Fig. 9, where the estimator receives twice as many measurements - from time $t=0 \ldots 50$ - before beginning prediction. The predictions remain synchronized with the actual system for a long period of time because errors in the frequency estimates are very small at the time measurements are halted.

\section{SUMMARY}

This paper has described a methodology for estimating structural vibrations using range images generated from raster scanning sensors. The method uses three distinct parts and exploits a key feature of space applications, which is that the dynamics of objects in space are well-modeled. This feature enables an estimator design that is both accurate and robust to the challenging sensing conditions found in space

A modal decomposition method was developed to handle partial and non-uniform visual sampling of a vibrating structure. This paper has augmented previous works by considering non-trivial effects introduced when using asynchronous raster-scanning sensors. Kalman filtering was shown to improve the coarse estimates provided by modal decomposition, and shape estimation then involved simply a modal reconstruction using the estimated modal coefficients.

Experiments were conducted using emulated space structures and sensors to study feasibility and practical implementation challenges. This work demonstrates that modal amplitude, phase, frequency, and damping can all be estimated accurately and efficiently using a single sensor viewing only a small portion of the structure. The results of these experiments have validated numerous simulation results reported in previous works and lend support for the use of this method in future space robotic systems.

\section{ACKNOWLEDGMENT}

The authors would like to acknowledge Satoshi Suzuki, Kazuyuki Inaba, and Dr. Tatsuya Endo for the design and fabrication of the experimental system and their valuable assistance while conducting experiments; and Dr. Yoshiaki Ohkami, Dr. Mitsushige Oda, and Dr. Karl Iagnemma for helpful feedback during the development of this work.

\section{REFERENCES}

[1] Mangalgiri, V. "Analysis for the Robotic Assembly of Large Flexible Space Structures.” MS Thesis, Dept. of Mech. Eng., MIT, 2004.

[2] Staritz, P.J., S. Skaff, C. Urmson, and W. Whittaker. "Skyworker: A Robot for Assembly, Inspection and Maintenance of Large Scale Orbital Facilities." Proc. 2001 IEEE Int. Conf. on Robotics and Automation (ICRA 2001), Seoul, Korea, pp. 4180-4185, May 2001.

[3] Ueno, H., T. Nishimaki, M. Oda, and N. Inaba. "Autonomous Cooperative Robots for Space Structure Assembly and Maintenance." Proc. 7th Int. Symp. on Artificial Intelligence, Robotics, and Automation in Space: i-SAIRAS 2003, NARA, Japan, May 2003.

[4] Mankins, J.C. "A Fresh Look at Space Solar Power: New Architectures, Concepts and Technologies." Proc. 38th Int. Astronautical Federation Conference, 1997.

[5] Oda, M., H. Ueno, and M. Mori. "Study of the Solar Power Satellite in NASDA." Proc. 7th Int. Symp. on Artificial Intelligence, Robotics, and Automation in Space: i-SAIRAS 2003, NARA, Japan, May 2003.

[6] Maghami, P.G. and S.M. Joshi. "Sensor/Actuator Placement for Flexible Space Structures." IEEE Trans. on Aerospace and Electronic Systems, Vol. 29, No. 2, pp. 345-351, April 1993

[7] Zhang, H.-H. and H.-X. Wu. "Identifiability of Flexible Space Structures." Proc. IEEE Conf. on Decision and Control, Vol. 2, pp. 1488-1493, Dec. 1993.

[8] Stieber, M.E., E.M. Petriu, and G. Vukovich. "Systematic Design of Instrumentation Architecture for Control of Mechanical Systems." IEEE Trans. on Instrument. and Meas., Vol. 45, No. 2, pp. 406-412, April 1996.

[9] Scattolini, R. and N. Cattane. "Detection of Sensor Faults in a Large Flexible Structure." IEE Proc. Control Theory and Applications, Vol. 146, No. 5, pp. 383-388, Sept. 1999.

[10] Vergauwen, M., M. Pollefeys, and L.J. Van Gool. "A Stereo-vision System for Support of Planetary Surface Exploration." Machine Vision and Applications, Vol. 14, No. 1, pp. 5-14, 2003.

[11] Wakabayashi, Y., Y. Ohkami, M. Miyata, T. Adachi, and T. Iijima. "A Compact Laser Range Finder for Space Applications." Proc. SPIE, Vol. 3714: Enabling Photonic Technologies for Aerospace Applications, A.R. Pirich, E.W. Taylor, eds., pp. 131-138, 1999.

[12] Jenkin, M. and P. Jasiobedzki. "Computation of Stereo Disparity for Space Materials." Proc. IEEE Int. Conf. on Intell. Robots and Sys. (IROS), 1998.

[13] Lichter, M.D. "Shape, Motion, and Inertial Parameter Estimation of Space Objects using Teams of Cooperative Vision Sensors." PhD Thesis, Dept. of Mech. Eng., MIT, Sept. 2004.

[14] Lichter, M.D., and S. Dubowsky. "Shape, Motion, and Parameter Estimation of Large Flexible Space Structures using Range Images." Proc. IEEE Int. Conf. on Robotics and Automation, Barcelona, Spain, April 2005.

[15] Julier, S.J., and J.K. Uhlmann. "A New Extension of the Kalman Filter to Nonlinear Systems." Proc. AeroSense: The 11th Int. Symp. on Aerospace/ Defense Sensing, Simulation and Controls, Orlando, Florida, 1997. 